\title{
Strain-specific transcriptional and posttranscriptional regulation of heat-labile toxin expression by enterotoxigenic Escherichia coli
}

\author{
Juliana Falcão Rodrigues ${ }^{1,2}$ - Rogério Ferreira Lourenço ${ }^{1,3}$ - Denicar Lina Nascimento Fabris Maeda ${ }^{1,2,4}$. \\ Mariana de Jesus Cintra ${ }^{1}$ - Naomi Nakao ${ }^{1}$ - Camila Mathias-Santos ${ }^{1,5}$ • Wilson Barros Luiz ${ }^{1,6}$. \\ Luís Carlos de Souza Ferreira ${ }^{1}$
}

Received: 22 July 2019 / Accepted: 18 January 2020 / Published online: 3 February 2020

(C) Sociedade Brasileira de Microbiologia 2020

\begin{abstract}
Enterotoxigenic Escherichia coli (ETEC) represents one of the most important etiological agents of diarrhea in developing countries and characteristically produces at least one of two enterotoxins: heat-labile toxin (LT) and heat-stable toxin (ST). It has been previously shown that the production and release of LT by human-derived ETEC strains are variable. Although the natural genetic polymorphisms of regulatory sequences of LT-encoding (eltAB) genes may explain the variable production of LT, the knowledge of the transcriptional and posttranscriptional aspects affecting LT expression among ETEC strains is not clear. To further understand the factors affecting LT expression, we evaluated the impact of the natural polymorphism in noncoding regulatory sequences of eltAB among clinically derived ETEC strains. Sequence analyses of seven clinically derived strains and the reference strain $\mathrm{H} 10407$ revealed polymorphic sites at both the promoter and upstream regions of the elt $A B$ operon. Operon fusion assays with GFP revealed that specific nucleotide changes in the Pribnow box reduce elt $A B$ transcription. Nonetheless, the total amounts of LT produced by the tested ETEC strains did not strictly correspond to the detected LTspecific mRNA levels. Indeed, the stability of LT varied according to the tested strain, indicating the presence of posttranscriptional mechanisms affecting LT expression. Taken together, our results indicate that the production of LT is a strain-specific process and involves transcriptional and posttranscriptional mechanisms that regulate the final amount of toxin produced and released by specific strains.
\end{abstract}

Keywords Heat-labile toxin · Enterotoxigenic Escherichia coli · Biodiversity · LT-specific mRNA · Transcriptional and posttranscriptional mechanisms

Commentary This manuscript was edited for proper English language, grammar, punctuation, spelling, and overall style by English-speaking editors at American Journal Experts in July 16, 2019 (certificate verification key: 8CBF-A585-44FF-00CF-5F84).

Electronic supplementary material The online version of this article (https://doi.org/10.1007/s42770-020-00231-2) contains supplementary material, which is available to authorized users.

Juliana Falcão Rodrigues

julifalcao_24@hotmail.com

$\triangle$ Luís Carlos de Souza Ferreira

lcsf@usp.br

1 Vaccine Development Laboratory, Department of Microbiology, Institute of Biomedical Sciences, University of São Paulo, Av. Prof. Lineu Prestes, 1374, Cidade Universitária, São Paulo, SP 05508-900, Brazil

2 Department of Parasitology, Institute of Biomedical Sciences, University of São Paulo, São Paulo, Brazil
3 Institute of Biology, The State University of Campinas, São Paulo, Brazil

4 Department of Pediatrics, University of Virginia, Charlottesville, VA, USA

5 Technical-Scientific Police Superintendency, São Paulo State, Criminalistic Institute, São Paulo, Brazil

6 Department of Biological Sciences, State University of Santa Cruz, Ilhéus, Bahia, Brazil 


\section{Introduction}

Diarrhea represents the third leading cause of child morbidity and mortality in the world, with serious impacts on public health policies and economies, particularly of lower-income countries [1]. Enterotoxigenic Escherichia coli (ETEC) remains an important etiological agent of diarrheal illness in childhood and is the most common cause of traveler's diarrhea [1-4]. The disease developed by ETEC strains requires the production of colonization factors ( $\mathrm{CFs}$ ), responsible for bacterial adhesion to enterocytes, as well as heat-stable toxins (ST) and/or heat-labile toxins (LT) [5, 6]. LT alone, or in combination with ST, is expressed by more than half of the ETEC isolates [7, 8]. Once produced and released into the small intestine, LT binds to host cells via the pentameric B subunit, which leads to increased intracellular cyclic adenosine monophosphate (cAMP) levels due to indirect enzymatic activity of the A subunit, causing water/electrolyte losses and, thereby, watery diarrhea of different severities $[5,6]$.

Although clonal and stable lineages of ETEC are spread worldwide, epidemiological studies have revealed high genetic and phenotypic diversity among ETEC strains regarding serotypes, virulence features, mainly CFs and LT types, and genotypes disclosed by molecular approaches [7, 9-14]. Our group previously showed the occurrence of 16 LT types among ETEC strains derived from asymptomatic and diarrheic Brazilian children [11]. These LT types were grouped into four phylogenetic clusters, two of which (A and D) comprised the majority of the variants closely related to the types LT1 and LT2, respectively. More recently, these results were confirmed by further studies, and twelve additional LT variants were found among clinical strains isolated from diverse geographic areas [12]. Genetic polymorphisms in noncoding regulatory sequences and in LT-structural genes have also been ascribed to different LT types [11, 12, 14]. However, considering the data available in the literature, thus far it has not been possible to correlate the ability to express LT and the natural genetic polymorphisms detected among different LT types.

LT expression and secretion are also variable traits observed among ETEC strains [12,15-17]. The amount of toxin secreted by strains producing only LT correlates with the severity of diarrhea in animal models and depends on the capability of the bacteria to produce and release the toxin [15]. LT expression is affected by different growth conditions, such as temperature, $\mathrm{pH}$, osmolarity, and the presence of glucose, which indicates the presence of diverse regulation mechanisms $[18,19]$.

The A and B subunits of LT are encoded by the eltA and elt $B$ genes, which are under the control of a single promoter and a transcriptional terminator [20-22]. LT production is repressed by heat-stable nucleoid-structural (H-NS) proteins, which bind a region of DNA near the transcriptional start site and a site at the end of the eltA gene [22]. Under environmental changes, particularly at $37^{\circ} \mathrm{C}$, the DNA-H-NS complex is destabilized, allowing the binding of RNA polymerase, leading to the synthesis of elt $A B$ polycistronic mRNA [22, 23]. In addition, it was reported that the cAMP receptor protein (CRP) negatively regulates LT expression, but there are doubts about the direct binding of this protein to the elt $A B$ regulatory region $[24,25]$. Posttranslational regulation of LT expression has also been reported, but the available evidence is weak [20, $21,26]$.

In the present study, we demonstrated that specific nucleotide changes at the promoter region of the elt $A B$ operon impact LT expression among clinically derived ETEC strains of different phylogenetic clusters. In addition, our results revealed that LT stability is affected by posttranscriptional mechanisms, which demonstrate that intrinsic transcriptional and posttranscriptional factors contribute to the strain-specific LT expression observed among ETEC strains.

\section{Materials and methods}

\section{Bacterial strains and growth conditions}

The LT-producing ETEC strains listed in Table 1 were previously characterized regarding genetic and phenotypic features $[10,11,15]$. The H10407 $\left(\mathrm{LT}^{+} \mathrm{ST}^{+} \mathrm{CFA}-\mathrm{I}^{+}\right)$is the most studied ETEC strain, particularly in regard to sequencing data and regulation mechanism of the elt $A B$ operon, and, therefore, it was used as a reference strain. The H10407 strain was kindly provided by Ann-Mari Svennerholm (University of Gothenburg, Gothenburg, Sweden). The BL21 competent $E$. coli were transformed with plasmids harboring the transcriptional fusions or with a control plasmid, generating the recombinant strains. The bacterial strains were cultivated in CA-YE medium ( $2 \%$ casamino acids, $0.6 \%$ yeast extract, $43 \mathrm{mM} \mathrm{NaCl}, 38 \mathrm{mM} \mathrm{K}_{2} \mathrm{HPO}_{4}$, and $0.1 \%$ trace salt solution consisting of $203 \mathrm{mM} \mathrm{MgSO}_{4}$, $25 \mathrm{mM} \mathrm{MnCl}_{2}$, and $18 \mathrm{mM} \mathrm{FeCl}_{3} ; \mathrm{pH}=8.4$ ) at $37^{\circ} \mathrm{C}$ and $200 \mathrm{rpm}[27]$.

\section{Sequencing and phylogenetic analyses}

The DNA sequences upstream of the translation start codon of the eltA gene, comprising 334 nucleotides, were amplified by PCR using genomic DNA from ETEC strains and sequenced by the Sanger method (ABI 3100 sequencer, Big Dye version 1, PerkinElmer Applied Biosystems) as previously described [11]. The forward and reverse primers for PCR and sequencing were constructed based on the sequence of the elt $A B$ operon from plasmid p666 (GenBank: FN649417.1), and their sequences are described in Table 2. Representative nucleotide sequences for each polymorphic group were deposited in 
Table 1 Phenotypic and genotypic features of LT-producing ETEC strains analyzed in the present study

\begin{tabular}{|c|c|c|c|c|c|c|}
\hline \multirow[t]{2}{*}{ Strain } & \multirow[t]{2}{*}{ Source $^{a}$} & \multirow[t]{2}{*}{ Serotype } & \multirow[t]{2}{*}{ LT Type } & \multirow[t]{2}{*}{ LT Group $^{c}$} & \multicolumn{2}{|c|}{ eltAB promoter polymorphisms } \\
\hline & & & & & -14 & -7 \\
\hline H10407 & $\mathrm{DC}$ & O78:H11 & LT1 & A, I & G & $\mathbf{C}$ \\
\hline $\begin{array}{l}136 \mathrm{I} \\
136 \mathrm{III}\end{array}$ & $\begin{array}{l}\mathrm{DC} \\
\mathrm{DC}\end{array}$ & $\begin{array}{l}\text { O88:H25 } \\
\text { O88:H25 }\end{array}$ & $\begin{array}{l}\mathrm{LT}^{b}{ }^{b} \\
\mathrm{LT}^{3}\end{array}$ & C, III & G & $\mathbf{T}$ \\
\hline $136 \mathrm{II}$ & DC & O88:H25 & LT5 & & & \\
\hline $\begin{array}{l}36 \mathrm{III} \\
36 \mathrm{IV}\end{array}$ & $\begin{array}{l}\text { DC } \\
\text { DC }\end{array}$ & $\begin{array}{l}\mathrm{O} 159: \mathrm{H} 21 \\
\mathrm{O} 159: \mathrm{H} 21\end{array}$ & $\begin{array}{l}\text { LT2 } \\
\text { LT2 }\end{array}$ & $\mathrm{D}, \mathrm{II}$ & $\mathbf{T}$ & $\mathbf{T}$ \\
\hline $187 \mathrm{~V}$ & DC & O159:H21 & LT2 & & & \\
\hline $63 \mathrm{~V}$ & $\mathrm{AC}$ & O159:H17 & LT14 & & & \\
\hline
\end{tabular}

${ }^{\text {a }} A C$ asymptomatic child, $D C$ diarrheic child

${ }^{\mathrm{b}}$ LT variants with (LT3) or without (LT5) the polymorphic site R13H in the B subunit

${ }^{\mathrm{c}}$ Phylogenetic relationship of the LT-I types according to Lasaro et al. (2008) and Joffré et al. (2015)

${ }^{\mathrm{d}}$ Polymorphisms in the eltAB promoter region at the positions -14 and -7 , according to the transcription start site, using $\mathrm{H} 10407$ as reference ETEC

GenBank: MN163050 (136I), MN163051 (36III), MN163052 (187 V). The phylogenetic relationship among the ETEC strains based on the sequenced regulatory nucleotides was represented by a rooted tree generated using the ClustalW program and neighbor-joining methods.

\section{Description of the eltAB-gfp transcriptional fusions and GFP detection assay}

The eltAB operon regions from UP elements to the start codon of translation (position -48 to +58 according to the transcription start), harboring the elt $A B$ promoter observed in the tested ETEC strains, were genetically fused to the DNA sequence encoding the green fluorescent protein (GFP) (GenBank number: KF410615.1). Three eltAB promoter$g f p$ gene transcriptional fusions were drawn regarding the polymorphisms observed in the elt $A B$ promoters of the ETEC strains using the eltAB operon from H10407 as a

Table 2 Oligonucleotides used in the present study

\begin{tabular}{ll}
\hline Oligonucleotide & Sequence \\
\hline FwRTrpoA & * \\
RvRTrpoA & 5'-AAGCTGGTCATCGAAATGGA-3' \\
FwRTeltB & 5'-GCCGCACGACGAATCG-3' \\
RvRTeltB & 5'-GGCAGGCAAAAGAGAAATGG-3' \\
FwCRPKpnI ${ }^{* *}$ & 5'-CGGGACTTCGACCTGAAATG-3' \\
RvPromoterelt $^{* *}$ & 5'-GGTGGGTACCTTCTGGTGCTC-3' \\
\hline
\end{tabular}

* Oligonucleotides labeled as RT were used for real-time PCR. FW and Rv denote forward and reverse, respectively. The target transcripts for RT$\mathrm{PCR}$ are indicated in the oligonucleotide names

** Oligonucleotide used for sequencing of the region upstream of the etxA gene, corresponding to 334 nucleotides. Underlined nucleotides indicate the $K p n I$ restriction site reference (Table 1$)$. The $\mathrm{pBSK}(+)$ vectors harboring the transcriptional fusions were manufactured by the Biomatik Company (Wilmington, Delaware, USA). A plasmid containing the $g f p$ gene under the control of the lepA promoter was used as a positive control for GFP production. The BL21 E. coli strains harboring the plasmids (recombinant strains) or not (mock) were grown in CA-YE medium for $6 \mathrm{~h}$ at $37^{\circ} \mathrm{C}$ and $200 \mathrm{rpm}$. Culture aliquots were centrifuged, and the cell sediments were resuspended in phosphatebuffered saline (PBS) 1x, generating bacterial suspensions equivalent to an $\mathrm{OD}_{600 \mathrm{~nm}}$ of 5 . The samples $(100 \mu \mathrm{l} / \mathrm{well})$ were transferred to a microtiter plate in triplicate, and the green fluorescence was detected with excitation at $490 \mathrm{~nm}$ and emission at 510-570 $\mathrm{nm}$ in a fluorometer (GlomaxMulti Detection System, Promega). The results were expressed as fluorescence arbitrary units.

\section{RNA extraction and RT-PCR}

For RNA isolation, ETEC strains were grown in CA-YE medium to an $\mathrm{OD}_{600 \mathrm{~nm}}$ of 5.0. Cells were harvested by centrifugation at $16,000 \mathrm{x}$ g for $1 \mathrm{~min}$, and total RNA was extracted by the Trizol method (Invitrogen). RNA samples were treated with DNase I (Fermentas) and tested for the absence of DNA contamination by PCR. RNA quality was evaluated by agarose/formaldehyde gel electrophoresis, and the yield was determined by NanoDrop 2000 (Thermo Fisher Scientific). Reverse transcription was performed using $2.5 \mu \mathrm{g}$ of RNA, 200 U of RevertAid Reverse Transcriptase (Fermentas), and $200 \mathrm{ng}$ of random hexamer primer (Fermentas). Quantitative PCR amplification of the resulting cDNA was carried out with Maxima SYBR Green/ROX qPCR Master Mix (Fermentas) and $0.4 \mu \mathrm{M}$ oligonucleotides specific for each gene (Table 2). Primers were designed using the Primer Express Software (Applied Biosystems), and their sequences are described in 
Table 2. The results obtained with the eltAB primers were normalized using the rpoA gene as the endogenous control, which was shown to be constant in the samples analyzed. In addition, efficiency analysis of the primers was tested using different amounts of elt $A B$-specific cDNA into the real-time PCR reactions. The rpoA gene-specific primers resulted in efficient amplification according to Ct-based standard curve analysis (equation $=-0.9339 \times+17.269 ; R^{2}=0.9990$ ) . Relative expression levels were calculated using the $2-\Delta \Delta C T$ method [28].

\section{LT production assay}

Measurement of the cell-associated and secreted LT levels among different ETEC strains was based on previously described protocols $[15,19]$. The culture samples of the reference strain $\mathrm{H} 10407$ were adjusted to an $\mathrm{OD}_{600 \mathrm{~nm}}$ equal to 0.1 and allowed to grow in CA-YE medium for $6 \mathrm{~h}$ until an $\mathrm{OD}_{600}$ $\mathrm{nm}$ is equivalent to approximately 5.0 or overnight cultures $(18 \mathrm{~h})$. The cell samples were adjusted to an $\mathrm{OD}_{600 \mathrm{~nm}}$ equal to 1 or 5 , with samples harvested after $6 \mathrm{~h}$, or to an $\mathrm{OD}_{600 \mathrm{~nm}}$ of 14 , with samples cultivated overnight. After cell lysis, culture supernatants and debris-free extracts of the bacterial pellets were recovered and kept at $4{ }^{\circ} \mathrm{C}$ before LT quantification $(\mathrm{ng} / \mathrm{ml})$ by ELISA. Alternatively, bacterial strains were cultivated for $6 \mathrm{~h}$, processed without normalizing the culture optical density, and the LT concentrations were adjusted according to the total protein content, which was determined using the Bradford assay. The last protocol allowed us to normalize LT concentration both in the bacterial cell extracts and supernatants and generate LT values from cell extracts similar to those obtained with cellular density adjustments equivalent to 5 (Fig. S1, Supplementary Material). For routine analyses of LT expression in the tested ETEC strains, we applied the protocol based on the normalization of LT expression by total protein content. The total LT production for each bacterial strain was calculated according to the following formula: secreted LT + cell-associated LT $(\mathrm{ng} / \mathrm{ml}) /$ total protein in the supernatant + cell-associated total protein $(\mathrm{mg} / \mathrm{ml})$, and the resulting values were expressed by ng of $\mathrm{LT} / \mathrm{mg}$ of total protein.

\section{LT and total bacterial protein quantifications}

Quantification of LT in the bacterial fractions was performed by capture ELISA as previously described [15]. Briefly, the microtiter plates (Nunc Maxisorp-Thermo Fisher Scientific, Waltham, Massachusetts, USA) were coated with rabbit anticholera toxin serum (titer equal to $1 \times 10^{6}$ ) diluted to $1: 1000$ in PBS 1x. After overnight incubation, twofold serial dilutions of the samples were placed on the plates, and the captured LT was detected by serial exposure to mouse anti-LT serum (titer $=5 \times 10^{4}$ ) and horseradish peroxidase-conjugated goat anti-mouse IgG antibodies (Sigma-Aldrich, St. Louis, MO, USA) diluted to 1:2500 and 1:5000, respectively. The reactions were read at $492 \mathrm{~nm}$ in an Epoch $^{\mathrm{TM}}$ Multi-Volume Spectrophotometer (BioTek Instruments, Vermont, USA). The Bradford assay was carried out to measure total protein in the bacterial samples according to the manufacturer's instructions (Coomassie Plus Protein Assay Kit, Thermo Fisher Scientific). Standard curves (regression analysis with $\mathrm{R}^{2}>$ 0.98 ), generated with the absorbance values versus concentrations of purified LT or bovine serum albumin (Pierce-Thermo Fisher Scientific), were used to determine LT and total protein concentrations, respectively, in the samples. Purified LT was obtained as previously reported [15].

\section{LT stability assay}

Determination of the LT stability in cell cultures of different ETEC strains was based on a protein synthesis inhibition assay using chloramphenicol [29]. ETEC strains were grown in CA-YE broth for $6 \mathrm{~h}$, and then the cultures were supplemented with chloramphenicol $(100 \mu \mathrm{g} / \mathrm{ml})$. Alternatively, the bacterial cultures were supplemented with chloramphenicol $(100 \mu \mathrm{g} / \mathrm{ml})$ and a protease inhibitor cocktail according to the manufacturer's instructions (ProteoBlock ${ }^{\mathrm{TM}}$ Protease Inhibitor Cocktail, Fermentas, Thermo Fisher Scientific). Aliquots (5 ml) were collected at different times $(0,30,60$, and $90 \mathrm{~min}$ ), and the bacterial cells were sonicated to release the LT toxin. The LT concentration was measured and normalized according to the total protein concentration. The LT stability was determined according to the following formula: (LT concentration in the cultures after 30-90 $\mathrm{min} \times 100$ )/LT concentration at $0 \mathrm{~h}$. The resulting values were expressed as percentages.

\section{Statistical analyses}

All data were represented as arithmetic means $\pm \mathrm{SE}$ and analyzed for two-way ANOVA with Tukey's post hoc test using GraphPad Prism v5 software (GraphPad Software, Inc., La Jolla, CA).

\section{Results}

\section{Natural genetic polymorphism of eltAB regulatory sequences in ETEC strains}

Genetic polymorphisms have been reported in the promoter and upstream regions of the LT1 and LT2 eltAB operons in ETEC strains [20]. We sequenced the region encompassing 334 nucleotides upstream of the translational start codon of the eltA gene of 8 ETEC strains expressing 4 different serotypes and 5 different LT types (1,2,3,5, and 14), according to 
a previously reported classification based on the eltA $B$ gene sequences (Table 1) [11]. According to the Kyoto Encyclopedia of Genes and Genomes (KEGG) (PMID 10592173), this 334 bp region may also contain the promoter elements of the 2 open reading frames (ORFs) divergently transcribed to the elt $A B$ operon (Fig. 1A). The sequence of the ETEC reference strain H10407 was identical to that derived from plasmid p666 and previously deposited in GenBank (GenBank: FN649417.1). Altogether, seven polymorphic sites were found in the sequence upstream of the transcription start site: four were located at presumed nonregulatory DNA sequences (T-229C, A-195G, T-92C, $\mathrm{A}-78 \mathrm{G}$ ), two at the promoter region (spacer and Pribnow box: G-14T and C-7T, respectively), and one at the UP regulatory element (deletion at position -51 from start site of transcription) (Fig. 1A). Two of these polymorphic sites were not previously reported (A-78G and a deletion at position 51), and the A-78G polymorphic site was characteristic of strains belonging to the O88 serogroup that express LT3/LT5 types. In addition, no polymorphic sites were found at the

a

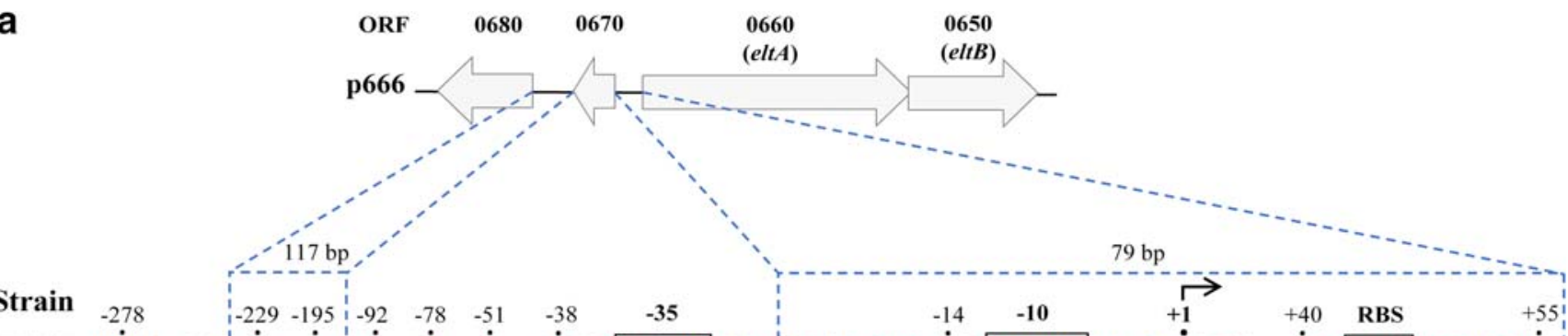

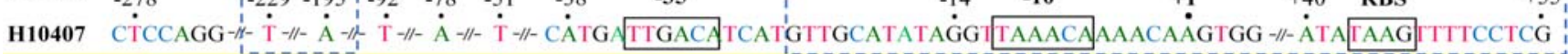

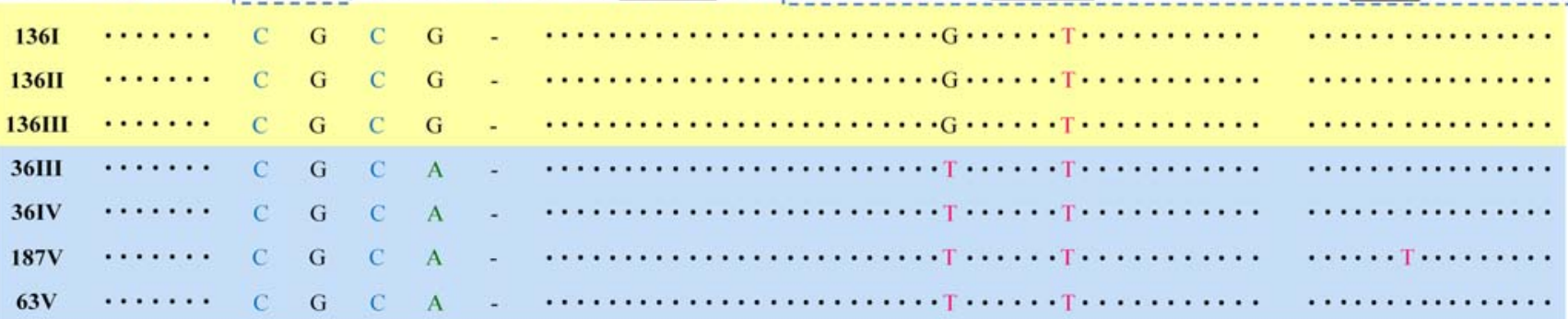

b

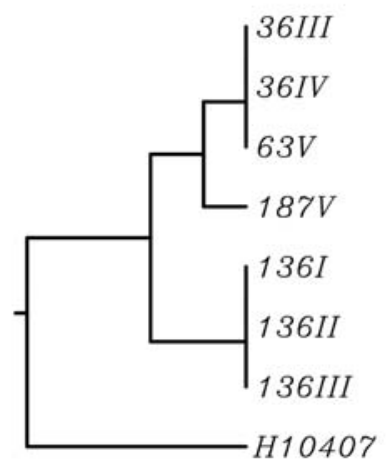

Fig. 1 Polymorphism in the eltAB promoter region among ETEC strains and the modifications on the promoter activity. (A) Alignment of the nucleotide sequences upstream of the translation start codon of the eltA genes from different ETEC strains obtained by Sanger sequencing. The transcription start site is indicated by an arrow as previously shown (Bodero and Munson, 2009). The -35 and -10 regions of the elt $A B$ promoter, as well as the ribosome binding site, are marked with boxes. The sequence from the H10407 strain used in our experiments, which is identical to that deposited in GenBank (FN649417.1), was used as a reference, and the nucleotide changes are indicated below for other ETEC strains. A schematic representation of the eltAB operon and genes divergently transcribed is shown above, and the intergenic

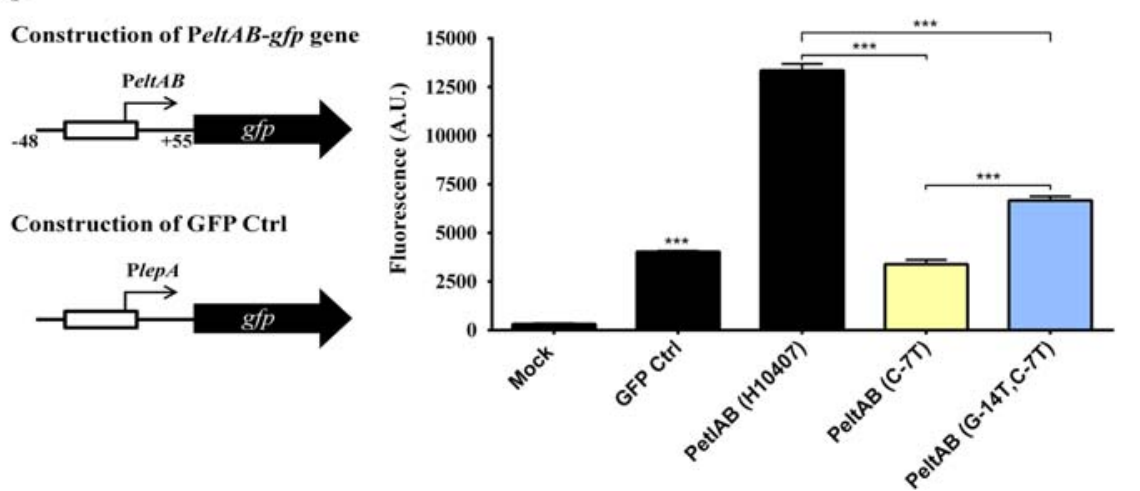

regions are highlighted in the sequence alignment. (B) Phylogenetic tree constructed from the sequences showed to Fig. 1A using the ClustalW program. (C) Left panel: schematic representation of the elt$g f p$ transcriptional fusions. The lepA-gfp fusion was used as a positive control for GFP expression in the laboratory E. coli strain (GFP Ctrl). (C) Right panel: measurement of GFP fluorescence. The original nontransformed $E$. coli strain (without $g f p$ gene) was used as negative control (Mock). The values represent the means \pm SE from three independent experiments. $* * * p<0.001$ and $* * * * p<0.0001$, significant differences with regard to the Mock group and between values indicated by brackets (two-way ANOVA with Tukey's test) 
previously reported CRP-binding sites [24]. Based on the determined elt $A B$ sequences, the tested strains were grouped into three clusters according to the serotypes and/or LT types (Fig. 1B and Table 1). As previously shown [14], our results confirmed the natural genetic polymorphism in the elt $A B$ transcriptional regulatory elements among LT1- and LT2producing ETEC strains and, for the first time, also among strains encoding LT3, LT5, and LT14 types.

\section{The impact of sequence polymorphism on the transcriptional activity of elt $A B$ genes}

The impact of the observed sequence polymorphisms on the transcriptional activity of the elt operon was determined after cloning a reporter gene downstream of the elt $A B$ promoter. The transcriptional fusions were constructed by combining the nucleotide changes at positions -14 and -7 (G-14 T and C-7 T): PeltAB(G-14, C-7), from the reference strain H10407, PeltAB(H10407), PeltAB(C-7 T), and PeltAB(G-14 T, C-7 T) (Fig. 1). The recombinant $E$. coli strains harboring the transcriptional fusions showed similar growth patterns to the nontransformed E. coli (data not shown). The presence of PeltAB(H10407) resulted in a two- to fourfold increase in GFP production compared to the expression observed with PeltAB(G-14 T, C-7 T) and PeltAB(C-7 T), respectively (Fig. 1C). These results indicate that the tested nucleotide changes affected the transcriptional activity of the $e l t A B$ genes. To confirm these findings, we monitored the production of LTspecific mRNA in different ETEC strains. In our experimental conditions, with the primers designed for $r p o A$, a housekeeping gene, and for elt B, as the target gene (FwRTeltB and RvRTeltB), we observed clear correlations among $C t$ values and the amounts of cDNA (Table 2, Fig. 2A and B). Based on this set of primers, the levels of eltB-specific transcripts in the $\mathrm{H} 10407$ strain were approximately 4 - and 12-fold higher than those expressed by the tested O159- or O88-associated ETEC strains, which harbor PeltAB(G-14 T, C-7 T) and PeltAB(C-7 T), respectively (Figs. 1 and 2C). Similar to the results generated with the operon fusion experiments, it was possible to demonstrate that the observed sequence polymorphisms in the promoter sequence affected the elt operon transcriptional regulation in all tested ETEC strains. Additionally, ETEC strains sharing
Fig. 2 LT-specific transcription among different ETEC strains. (A and $\mathbf{B})$ Evaluation of primer efficiency on the detection of eltB (A) and rpoA (B) expression. Different amounts of specific cDNA from H10407 ETEC were used for the RT-PCR. (C)

Expression of LT-specific mRNA measured by RT-PCR among ETEC strains harboring different elt $A B$ promoter regions: PeltAB(H10407) (white bars), PeltAB(G-14 T, C-7 T) (black bars), and Pelt $A B(\mathrm{C}-7 \mathrm{~T}$ ) (gray bars). The values represent the means \pm SE from two independent experiments. * $p<0.01$, significant difference with regard to the values represented by the gray bars, and $* * * p<0.001$, significant differences with regard to the values represented by the black and gray bars (two-way ANOVA with Tukey's test) a
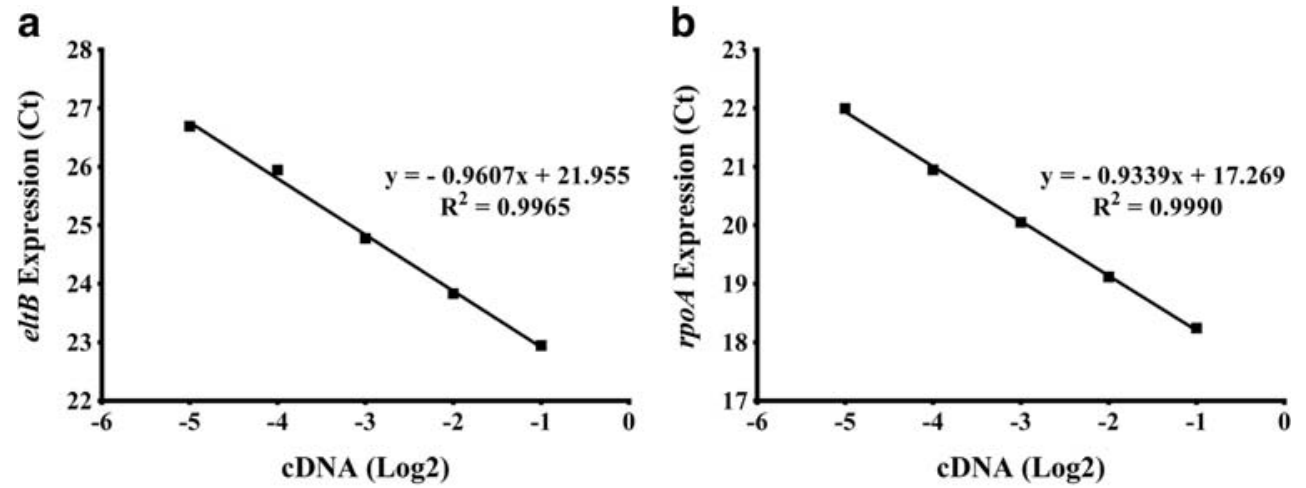

C

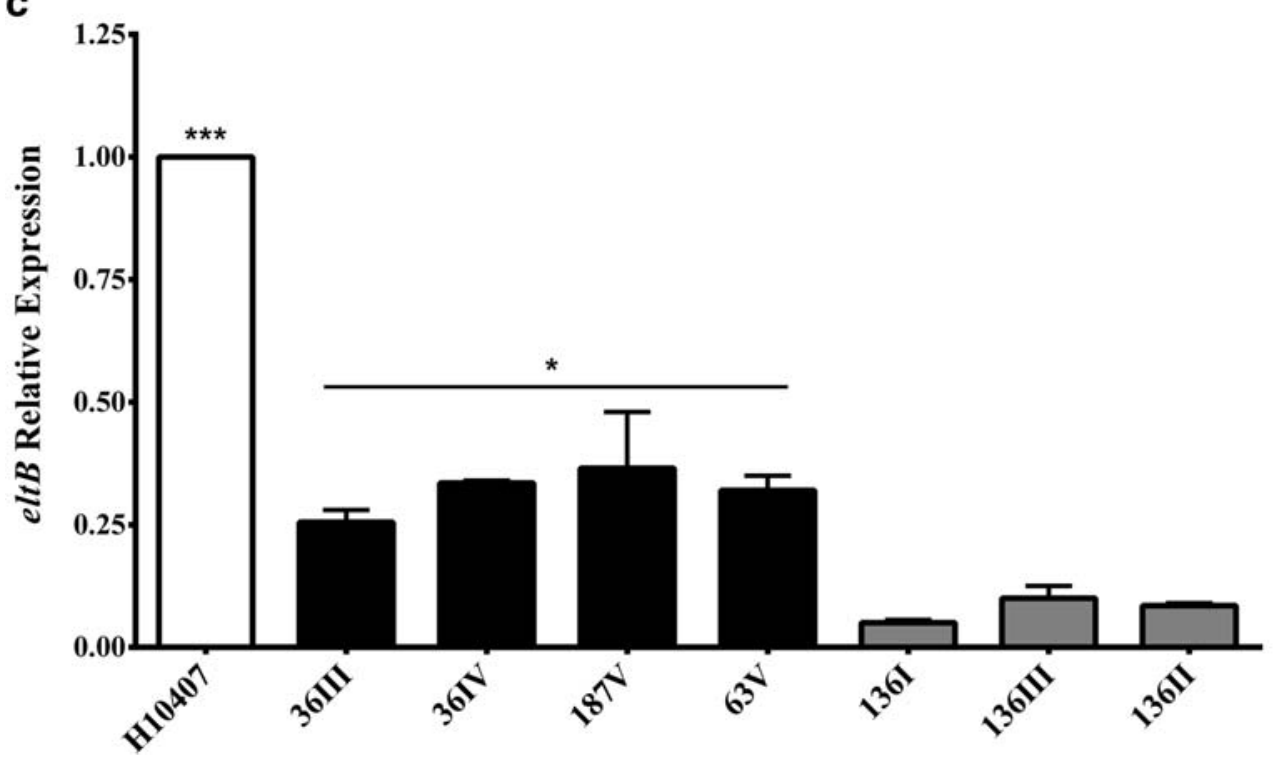


the same serotype/serogroup showed similar transcriptional regulation of the eltAB operon.

\section{Posttranscriptional factors affecting LT expression}

We also determined the action of posttranscriptional regulatory factors by measuring the amount and stability of LT produced by the different ETEC strains. For that purpose, we standardized the quantification using the total protein content present in both bacterial cell lysates and culture supernatants as a reference to measure the amount of cell-associated and secreted LT level (see Materials and Methods, Fig. S1, Supplementary Material). The total amount of LT produced by the tested ETEC strains ranged from 64 to $177 \mathrm{ng}$ of toxin/ mg of total protein, which included both cell-associated and secreted toxin (Fig. 3). Similar to the results based on the measurement of LT-specific mRNA levels, the H10407 strain expressed the highest LT concentrations among all tested ETEC strains (Figs. 2C and 3). Nonetheless, the amount of LT produced by H10407 [PeltAB(G-14, C-7)] did not correspond to the amount of transcript when compared with the O88:H25 strains [PeltAB(C-7 T)] and O159-H21/H17 strains $[$ PeltAB(G-14 T, C-7 T)]. This observation indicates that the amount of LT produced by ETEC strains is also regulated by posttranscriptional factors. To demonstrate the differential stability behavior of LT produced by different treatments, we measured the amount of cell-associated LT in bacterial cultures treated with a protein synthesis inhibitor. As observed in Fig. 4, the stability of LT produced by H10407 was significantly lower than those observed with the other tested ETEC strains (Fig. 4A-C). In addition, incubation of the cells in the presence of a protease inhibitor mixture recovered the stability of LT produced by the H10407 strain (Fig. 4D). Collectively, these results demonstrated that LT production by ETEC strains is affected both by transcriptional and posttranscriptional elements.

\section{Discussion}

ETEC strains have shown remarkable heterogeneity regarding genotypes, phenotypic and serological traits, as well as virulence factor profiles [7, 10, 13]. ETEC may also be distinguished according to the expressed LT type and amount of toxin produced and secreted $[11,12,15,30]$. In the present study, we demonstrated that the natural polymorphism in the elt $A B$ promoter region may lead to different amounts of transcripts. Additionally, we demonstrated that the final amount of LT produced by the ETEC strain may also be affected, as demonstrated with the reference strain H10407, by the presence of cellular proteases that reduced the stability of synthesized LT. Altogether, our results demonstrate that the genetic diversity observed among the eltAB operon of ETEC strains may impact, both at the transcriptional and posttranscriptional levels, the expression of LT.

Bacterial gene expression is controlled by RNA polymerase and regulatory proteins that are dependent on the promoter-sigma factor interaction [31]. We identified polymorphic sites at the eltAB promoter region from different ETEC strains expressing the main LT phylogenetic groups. These mutations were previously shown by Joffré and Sjöling (2016), but it was not possible to correlate them with distinct levels of eltAB gene-specific transcripts so far [14]. Here, we demonstrated differences in transcriptional activity according to the three clusters of mutations found in the promoters of the eltAB operon from the ETEC strains using GFP as a reporter gene. The data obtained with the promoter- $g f p$ gene fusions were correlated with the distinct patterns of specific mRNA expression exhibited by the strains. The presence of cytosine in the Pribnow box proved to be critical to better the performance of the promoter activity of the elt $A B$ operon since the transition of C-7 T diminished GFP and LT expression. Supporting these results, LT2 producers harboring the promoter PeltAB (C-7) [14] showed LT production profiles more similar to the $\mathrm{H} 10407$ reference strain [PeltAB(C-7)]
Fig. 3 Expression of LT by the different ETEC strains. Total LT production was measured by quantitative capture ELISA and normalized by the total protein content of each sample. The values represent the means $\pm \mathrm{SE}$ from three independent experiments. $* p<0.05$ and $* * p<0.01$, significant differences with regard to the $\mathrm{H} 10407$ strain (two-way ANOVA with Tukey's test)

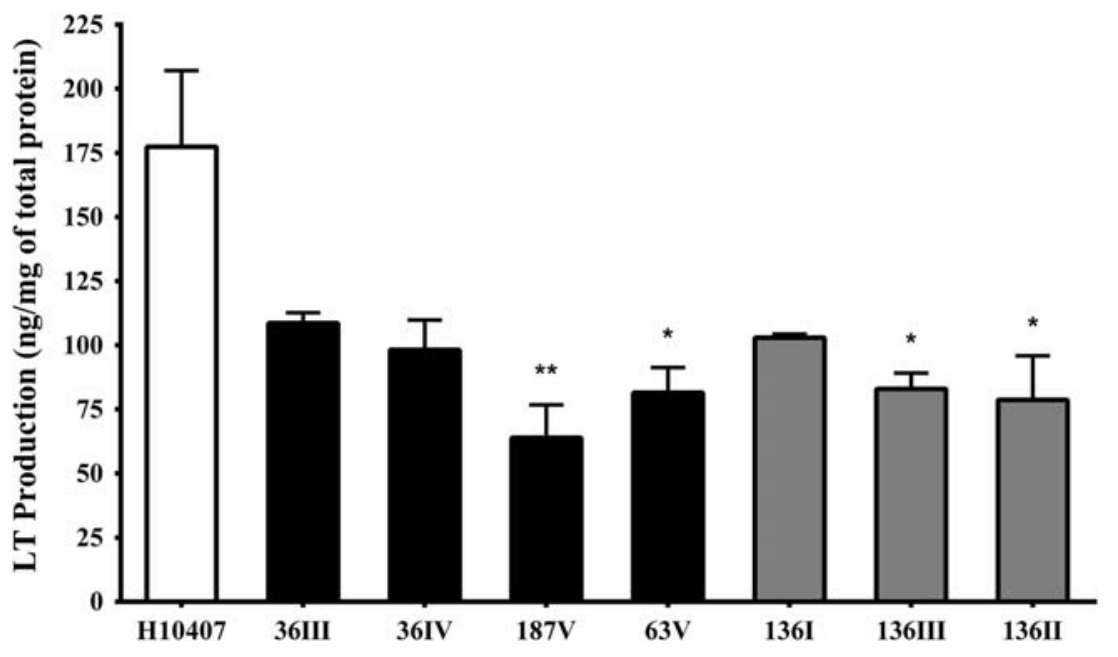



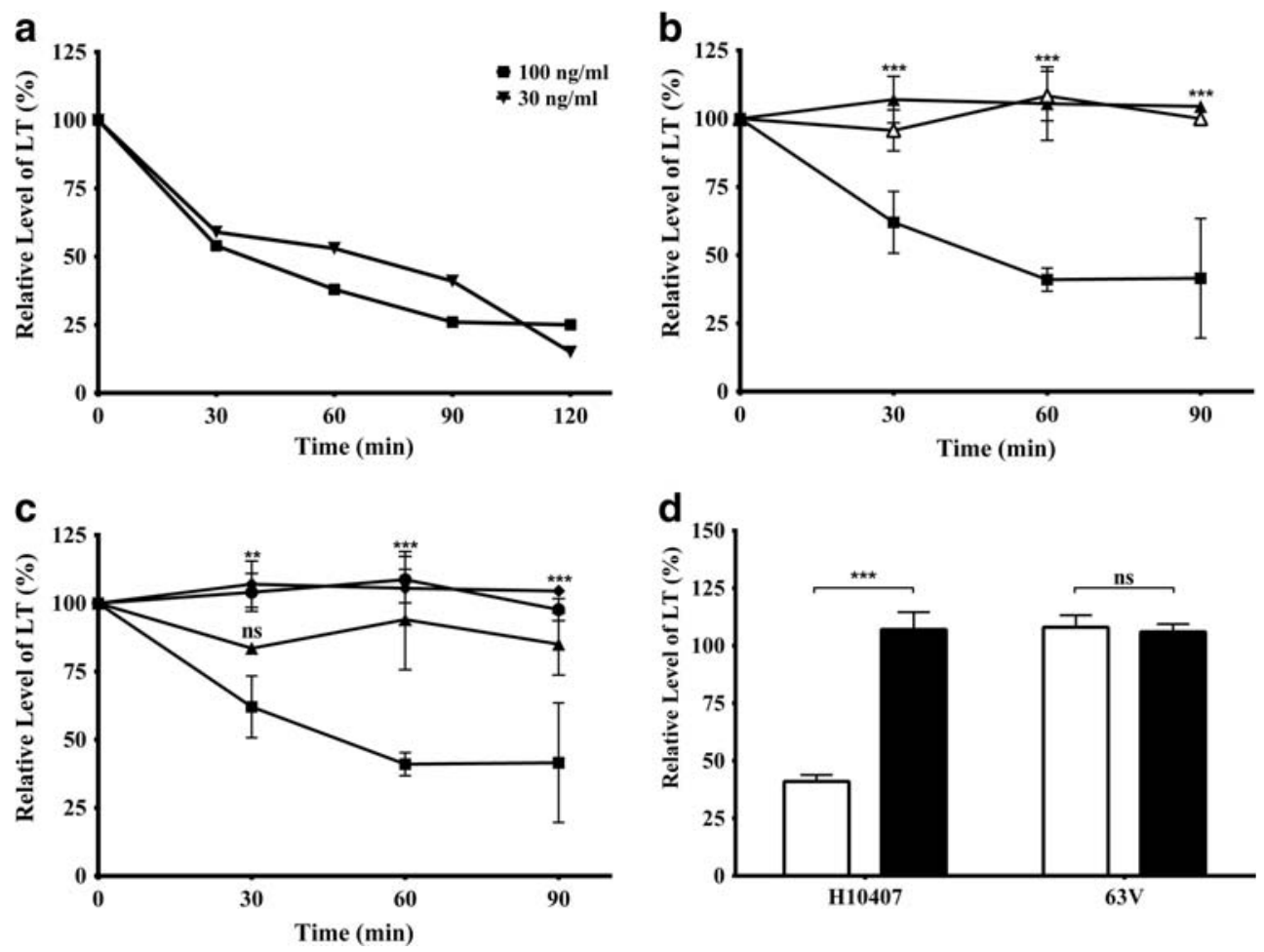

Fig. 4 Stability of LT produced by different ETEC strains. (A-D) Determination of LT stability among the tested ETEC strains. Chloramphenicol was used as a protein synthesis inhibitor at 30 (A) or $100 \mathrm{ng} / \mathrm{ml}$ (B-D), at 6-h culture time ( $0 \mathrm{~h}$ in Figs. A-C), in combination (D) or not (A-C) with protease inhibitor cocktail. (B) Evaluation of LT stability of ETEC strains expressing serogroup O159 and LT2 or LT14 in comparison with H10407. Symbols are represented as follows: (ש) H10407, ( $\Delta$ ) 36III, and ( $\Delta$ ) $63 \mathrm{~V}$. (C) Evaluation of LT stability of the ETEC strains expressing the serogroup O88 and LT3 or LT5 in

than the LT2-associated ETEC strains evaluated in the present study $[\mathrm{Pelt} A B(\mathrm{C}-7 \mathrm{~T})]$. In all allelic variants, the -10 region of the elt $A B$ operon remains a highly AT-rich promoter, which may not interfere with promoter opening. However, cytosine instead of thymine at the -7 position may increase recognition by sigma factor 70 and consequently may improve gene transcription. A similar behavior was observed when promoters that regulate genes belonging to the adaptive response to DNA alkylating agents were mutated [32]. Together, these results demonstrate that genetic polymorphisms in the promoter impact the transcriptional activity of the elt $A B$ operon and contribute to the diversity of LT expression in ETEC.

Although the C-7 $\mathrm{T}$ mutation accounts for the decreased expression of eltAB in the ETEC strain analyzed, we cannot state that this mutation reduced the affinity of the RNApolymerase holoenzyme for the -10 promoter element. We found two open reading frames (ORFs) divergently transcribed to the eltAB genes - ORFs 0680 and 0670 . Therefore, the promoter region of the 0670 and even 0680 could be located within the region encompassing -48 to + 55 relative to the transcription start site $(+1)$ of $e l t A B$, which was used in the $g f p$ gene constructions. By changing the

comparison with H10407. Symbols are represented as follows: (a) H10407, ( $\Delta$ ) 136I, (•) 136II, and ( $\bullet$ 136III. (D) The values correspond to the analysis carried out at $1 \mathrm{~h}$ after incubation with the inhibitors. The values represent the average $\pm \mathrm{SE}$ of the LT relative amounts $(\%)$ in comparison to those obtained before treatments for each strain. The assays were repeated three times. ${ }^{* *} p<0.01$ and $* * * p<0.001$, significant differences with regard to the H10407 strain (B-C) or as indicated by a bracket (D); $n s$ values without statistically significant differences (twoway ANOVA with Tukey's test)

occupancy of this region with transcriptional factors involved in expression of 0670 and 0680 , the C-7 T mutation could alter the accessibility of RNA-polymerase to the -10 promoter element of the eltAB operon instead of a direct effect of this mutation on the binding affinity of the enzyme for the promoter.

Single nucleotide polymorphisms (SNPs) were also observed in the UP element (deletion at position - 51) and region upstream from it among tested ETEC strains. Considering that deletion of the UP element did not interfere with eltAB transcription according to Yang and coworkers [22], the polymorphism in this region of the eltAB operon may not be responsible for the variability in the LT-specific mRNA levels among the ETEC strains tested in the present study. On the other hand, the four polymorphic sites upstream of the UP element could contribute to the elt $A B$-specific transcript levels. Additional analyses are required to evaluate the impact of SNPs upstream of the promoter on the diversity of LT expression among the ETEC strains.

Reports in the literature have also demonstrated modulation of LT expression in ETEC by global regulators such as HNS and CRP proteins [19, 22, 24, 25]. Although nucleotide 
substitutions at the H-NS- or CRP-binding sites as well as genes encoding each regulator are rarely found in bacteria sharing close phylogenetic relationships, such mutations may affect the activities of these regulators [33-35]. Data obtained here and by other researchers did not detect polymorphic sites at the CRP-binding sites previously described by Bodero and Munson (2009) [14, 24]. However, we found variability in the amount of LT produced in the presence or absence of glucose among ETEC strains, indicating that eltAB gene repression or activation by CRP is a strain-specific phenomenon (data not shown). Sahl and Rasko (2012) also observed diversity in the effect of glucose and bile salts on the expression of LT-encoding genes upon comparing H10407 and E24377A strains [36]. Together, these data demonstrate that ETEC strains may diverge regarding the elt operon regulation networking under different environmental stimulus. Additionally, our results are in accordance with the previous observation that CRP, in fact, does not bind to the eltAB operon and indirectly controls LT expression.

We did not observe a strict correlation between transcription levels of the eltAB operon and total amount of LT produced by the tested ETEC strains, indicating that posttranscriptional events were also regulating LT production. One polymorphism was found in the RBS of the eltB gene from H10407 and could contribute to compensation at the translation level of the strong transcriptional activity of the eltAB operon in comparison with other tested strains. However, additional experiments are required to confirm this observation. On the other hand, we consistently detected instability of H10407-derived LT (LT1) under culture growth conditions, which at least partially explains why H10407 [PeltAB(G-14, C-7)] expressed four to ten times more elt $A B$ transcripts but produced only approximately two times more LT than ETEC strains from serogroups $\mathrm{O} 88$ and $\mathrm{O} 159[\mathrm{PeltAB}(\mathrm{C}-7 \mathrm{~T})$ and PeltAB(G-14 T, C-7 T), respectively]. The stability of this protein was recovered under treatment with protease inhibitors, indicating strong control of LT production by the cellular protein degradation machinery in H10407. This susceptibility to proteolysis does not seem to be due to LT type and may be ascribed to specificities of the proteolytic machinery of H10407. The proteolytic routes have been reported in the literature particularly the control of expression and activity of virulence and physiology regulators in bacteria [37, 38]. However, proteolysis of LT as regulatory mechanism of expression has not been described previously. Collectively, the results showed in the present study demonstrate that LT production is regulated by both transcriptional and posttranscriptional mechanisms of protein regulation, allowing the control of overexpression of LT by some ETEC strains.

Despite the natural diversity of ETEC, clonal relationships among some strains have been demonstrated by phenotypic and genotypic methods [6, 7, 10, 13]. As such, ETEC strains sharing determined serotypes or serogroups have shown the same pattern of virulence factors; for example, the O6:H16 strains are $\mathrm{CFA}-\mathrm{II}^{+} \mathrm{LT}^{+} \mathrm{ST}^{+}[7]$. Here, we observed that strains sharing the same serogroup (O88 or O159) and expressing phylogenetically clustered LTs (LT3/LT5 or LT2/LT14, respectively) show similar patterns of LT production and the same allelic variant in the $\operatorname{etx} A B$ promoter $[\mathrm{Pelt} A B(\mathrm{C}-7 \mathrm{~T})$ or PeltAB(G-14 T, C-7 T), respectively] in comparison with reference ETEC H10407 [PeltAB(G-14, C-7)]. In fact, such lineages sharing the same serogroup present a close genetic relationship defined by the MLEE, PFGE, and RAPD profiles [10]. Additionally, our results together with those reported by Joffré and Sjöling (2016) [14] reveal that LT2-producing strains with different genetic backgrounds show distinct LT production profiles. In fact, previous reports at the global or local geographic scale have demonstrated the diversity of LT expression among ETEC strains, even among strains expressing the same LT type $[11,12,15]$.

Collectively, these findings suggest that the determined allelic variants of the elt $A B$ genes encoding phylogenetically related LTs may have been distributed by horizontal transfer to $E$. coli strains with different genetic backgrounds and that the production of the toxin would be impacted by specificities of the regulatory regions in the elt $A B$ operon and, particularly, by strain-specific characteristics of the bacterial cellular machinery. Considering that the level of LT production and secretion may contribute to the severity of diarrhea, further studies focused on the diversity of LT expression, and the virulence of the strain shall contribute to the design of more rational preventive and therapeutic approaches to control diarrhea associated with ETEC infection.

Funding information This study was supported by grants from Fundação de Amparo à Pesquisa do Estado de São Paulo (FAPESP grant numbers: 2016/20045-7, 04/02242-2), Conselho Nacional de Desenvolvimento Científico e Tecnológico (CNPq grant number: 509855/2010-5), and Coordenação de Aperfeiçoamento de Pessoal de Nível Superior (CAPES). We thank the helpful technical assistance of E. G. Martins.

\section{Compliance with ethical standards}

Conflict of interest On behalf of all authors, the corresponding author states that there is no conflict of interest.

\section{References}

1. Wang H, Naghavi M, Allen C et al (2016) Global, regional, and national life expectancy, all-cause mortality, and cause-specific mortality for 249 causes of death, 1980-2015: a systematic analysis for the global burden of disease study 2015. Lancet 388:14591544. https://doi.org/10.1016/S0140-6736(16)31012-1

2. Diemert DJ (2006) Prevention and self-treatment of traveler's diarrhea. Clin Microbiol Rev 19:583-594. https://doi.org/10.1128/ CMR.00052-05

3. Pires SM, Fischer-Walker CL, Lanata CF et al (2015) Aetiologyspecific estimates of the global and regional incidence and mortality 
of diarrhoeal diseases commonly transmitted through food. PLoS One 10:1-17. https://doi.org/10.1371/journal.pone.0142927

4. Bill F, Foundation MG (2017) Estimates of global, regional, and national morbidity, mortality, and aetiologies of diarrhoeal diseases: a systematic analysis for the global burden of disease study 2015. Lancet Infect Dis 17:909-948. https://doi.org/10.1016/S14733099(17)30276-1

5. Nataro JP, Kaper JB (1998) Diarrheagenic Escherichia coli. Clin Microbiol Rev 11:142-201 http://www.ncbi.nlm.nih.gov/pubmed/ 9457432

6. Gomes TAT, Elias WP, Scaletsky ICA, Guth BE, Rodrigues JF, Piazza RM, Ferreira LC, Martinez MB (2016) Diarrheagenic Escherichia coli. Braz J Microbiol 47:3-30. https://doi.org/10. 1016/j.bjm.2016.10.015

7. Wolf MK (1997) Occurrence, distribution, and associations of O and $\mathrm{H}$ serogroups, colonization factor antigens, and toxins of enterotoxigenic Escherichia coli. Clin Microbiol Rev 10:569-584

8. Qadri F, Svennerholm A-M, Faruque ASG, Sack RB (2005) Enterotoxigenic Escherichia coli in developing countries: epidemiology, microbiology, clinical features, treatment, and prevention. Clin Microbiol Rev 18:465-483. https://doi.org/10.1128/CMR.18. 3.465

9. Pacheco ABF, Guth BEC, Soares KCC, Nishimura L, De Almeida DF, Ferreira LCS (1997) Random amplification of polymorphic DNA reveals serotype-specific clonal clusters among enterotoxigenic Escherichia coli strains isolated from humans. J Clin Microbiol 35:1521-1525

10. Regua-Mangia AH, Guth BC, Da Costa Andrade JR et al (2004) Genotypic and phenotypic characterization of enterotoxigenic Escherichia coli (ETEC) strains isolated in Rio de Janeiro city, Brazil. FEMS Immunol Med Microbiol 40:155-162. https://doi. org/10.1016/S0928-8244(03)00308-0

11. Lasaro MA, Rodrigues JF, Mathias-Santos C, Guth BE, Balan A, Sbrogio-Almeida ME, Ferreira LC (2008) Genetic diversity of heatlabile toxin expressed by enterotoxigenic Escherichia coli strains isolated from humans. J Bacteriol 190:2400-2410. https://doi.org/ 10.1128/JB.00988-07

12. Joffré E, von Mentzer A, Abd El Ghany M et al (2015) Allele variants of enterotoxigenic Escherichia coli heat-labile toxin are globally transmitted and associated with colonization factors. J Bacteriol 197:392-403. https://doi.org/10.1128/jb.02050-14

13. Von Mentzer A, Connor TR, Wieler LH et al (2014) Identification of enterotoxigenic Escherichia coli (ETEC) clades with long-term global distribution. Nat Genet 46:1321-1326. https://doi.org/10. 1038/ng.3145

14. Joffré E, Sjöling Å (2016) The LT1 and LT2 variants of the enterotoxigenic Escherichia coli (ETEC) heat-labile toxin (LT) are associated with major ETEC lineages. Gut Microbes 7:75-81. https:// doi.org/10.1080/19490976.2015.1127480

15. Lasaro MAS, Rodrigues JF, Mathias-Santos C, Guth BE, RéguaMangia A, Piantino Ferreira AJ, Takagi M, Cabrera-Crespo J, Sbrogio-Almeida ME, de Souza Ferreira LC (2006) Production and release of heat-labile toxin by wild-type human-derived enterotoxigenic Escherichia coli. FEMS Immunol Med Microbiol 48: 123-131. https://doi.org/10.1111/j.1574-695X.2006.00134.x

16. Rocha LB, Ozaki CY, Horton DSPQ, Menezes CA, Silva A, Fernandes I, Magnoli FC, Vaz TM, Guth BE, Piazza RM (2013) Different assay conditions for detecting the production and release of heat-labile and heat-stable toxins in enterotoxigenic Escherichia coli isolates. Toxins 5:2384-2402. https://doi.org/10.3390/ toxins 5122384

17. Joffré E, von Mentzer A, Svennerholm AM, Sjöling Å (2016) Identification of new heat-stable (STa) enterotoxin allele variants produced by human enterotoxigenic Escherichia coli (ETEC). Int J Med Microbiol 306:586-594. https://doi.org/10.1016/j.ijmm.2016. 05.016
18. Hegde A, Bhat GK, Mallya S (2009) Effect of stress on production of heat labile enterotoxin by Escherichia coli. Indian J Med Microbiol 27:325-328. https://doi.org/10.4103/0255-0857.55446

19. Gonzales L, Ali ZB, Nygren E et al (2013) Alkaline pH is a signal for optimal production and secretion of the heat labile toxin, LT in enterotoxigenic Escherichia coli (ETEC). PLoS One 8:1-12. https://doi.org/10.1371/journal.pone.0074069

20. Yamamoto T, Yokota T (1983) Sequence of heat-labile enterotoxin of Escherichia coli pathogenic for humans. J Bacteriol 155:728733

21. Yamamotos T, Yokota T (1984) Primary structure of heat-labile enterotoxin produced by Escherichia coli pathogenic for humans. J Biol Chem 259:5037-5044

22. Yang J, Tauschek M, Strugnell R, Robins-Browne RM (2005) The H-NS protein represses transcription of the eltAB operon, which encodes heat-labile enterotoxin in enterotoxigenic Escherichia coli, by binding to regions downstream of the promoter. Microbiology 151:1199-1208. https://doi.org/10.1099/mic.0.27734-0

23. Trachman JD, Maas WK (1998) Temperature regulation of heatlabile enterotoxin (LT) synthesis in Escherichia coli is mediated by an interaction of H-NS protein with the LT A- subunit DNA. J Bacteriol 180:3715-3718

24. Bodero MD, Munson GP (2009) Cyclic AMP receptor proteindependent repression of heat-labile enterotoxin. Infect Immun 77: 791-798. https://doi.org/10.1128/IAI.00928-08

25. Haycocks JRJ, Sharma P, Stringer AM, Wade JT, Grainger DC (2015) The molecular basis for control of ETEC enterotoxin expression in response to environment and host. PLoS Pathog 11: e1004605. https://doi.org/10.1371/journal.ppat.1004605

26. Dorsey FC, Fischer JF, Fleckenstein JM (2006) Directed delivery of heat-labile enterotoxin by enterotoxigenic Escherichia coli. Cell Microbiol 8:1516-1527. https://doi.org/10.1111/j.1462-5822. 2006.00736.x

27. Evans DG, Silver RP, Evans DJ, Chase DG, Gorbach SL (1975) Plasmid controlled colonization factor associated with virulence in Escherichia coli enterotoxigenic for humans. Infect Immun 12: 656-667

28. Livak KJ, Schmittgen TD (2001) Analysis of relative gene expression data using real-time quantitative PCR and the 2- $\Delta \Delta \mathrm{CT}$ method. Methods 25:402-408. https://doi.org/10.1006/meth.2001.1262

29. Bacchus AN, Javor GT (1975) Stability of Escherichia coli membrane proteins during chloramphenicol treatment. Antimicrob Agents Chemother 8:387-389 http://www.ncbi.nlm.nih.gov/ entrez/query.fcgi?cmd=Retrieve $\& d b=$ PubMed $\& d o p t=$ Citation\&list uids $=1101826$

30. Sjöling Å, Qadri F, Nicklasson M, Begum YA, Wiklund G, Svennerholm AM (2006) In vivo expression of the heat stable (estA) and heat labile (eltB) toxin genes of enterotoxigenic Escherichia coli (ETEC). Microbes Infect 8:2797-2802. https:// doi.org/10.1016/j.micinf.2006.08.011

31. Lodish H, Berka A, Matsudaira P, Kaiser C, Krieger MSM (2008) Molecular cell biology. In: Lodish H (ed) Biologia Celular e molecular, 7th edn. Artmed, Guelph

32. Lacour S, Kolb A, Landini P (2003) Nucleotides from -16 to -12 determine specific promoter recognition by bacterial $\sigma \mathrm{S}-\mathrm{RNA}$ polymerase. J Biol Chem 278:37160-37168. https://doi.org/10.1074/ jbc.M305281200

33. Stoltzfus L, Wilcox G (1989) Effect of mutations in the cyclic AMP receptor protein-binding site on araBAD and araC expression. $\mathrm{J}$ Bacteriol 171:1178-1184. https://doi.org/10.1128/jb.171.2.11781184.1989

34. Ueguchi C, Suzuki T, Yoshida T, Tanaka KI, Mizuno T (1996) Systematic mutational analysis revealing the functional domain organization of Escherichia coli nucleoid protein H-NS. J Mol Biol 263:149-162. https://doi.org/10.1006/jmbi.1996.0566 
35. Hunt DM, Saldanha JW, Brennan JF, Benjamin P, Strom M, Cole JA, Spreadbury CL, Buxton RS (2008) Single nucleotide polymorphisms that cause structural changes in the cyclic AMP receptor protein transcriptional regulator of the tuberculosis vaccine strain Mycobacterium bovis BCG alter global gene expression without attenuating growth. Infect Immun 76:2227-2234. https://doi.org/ 10.1128/IAI.01410-07

36. Sahl JW, Rasko DA (2012) Analysis of global transcriptional profiles of enterotoxigenic Escherichia coli isolate E24377A. Infect Immun 80:1232-1242. https://doi.org/10.1128/IAI.06138-11
37. Gur E, Biran D, Ron EZ (2011) Regulated proteolysis in gramnegative bacteria-how and when? Nat Rev Microbiol 9:839-848. https://doi.org/10.1038/nrmicro2669

38. Frees D, Brøndsted L, Ingmer H (2013) Bacterial proteases and virulence. Subcell Biochem 66:161-192. https://doi.org/10.1007/ 978-94-007-5940-4_7

Publisher's note Springer Nature remains neutral with regard to jurisdictional claims in published maps and institutional affiliations. 